Cavendish-HEP-94/16

Liverpool-HEP-95/201

hep-ph/9504232

\title{
HERBVI - a program for simulation of baryon and lepton number violating processes"
}

\author{
M.J. Gibbsi and B.R. Webber \\ Cavendish Laboratory, University of Cambridge \\ Madingley Road, Cambridge CB3 0HE, U.K.
}

\begin{abstract}
We describe a Monte Carlo event generator for the simulation of baryon- and lepton-number violating processes at supercolliders. The package, HERBVI, is designed as a hard-process generator interfacing to the general hadronic event simulation program HERWIG. In view of the very high multiplicity of gauge bosons expected in such processes, particular attention is paid to the efficient generation of multiparticle phase space. The program also takes account of the expected colour structure of baryon-number violating vertices, which has important implications for the hadronization of the final state.
\end{abstract}

Submitted to Comp. Phys. Comm.

Cavendish-HEP-94/16

Liverpool-HEP-95/201

June 27, 2021

\footnotetext{
* Research supported in part by the UK Science and Engineering Research Council and by the EC Programme "Human Capital and Mobility", Network "Physics at High Energy Colliders", contract CHRXCT93-0357 (DG 12 COMA).

${ }^{\dagger}$ Current Address: Department of Physics, Oliver Lodge Laboratory, P.O. Box 147, Liverpool University, Liverpool L69 3BX.
} 


\section{Program summary}

Title of program: HERBVI

Program obtainable from: gibbs@dxcern.cern.ch

Prgramming language used: FORTRAN 77

Memory required: approx. 120 kbytes

Number of bits per word: 32

Subprograms used: HERWIG version 5.7 or later

Number of lines in distributed program: 4000

Keywords: baryon and lepton number violation, Monte Carlo techniques, high particle multiplicites

Nature of physical problem: simulation of baryon and lepton number violating processes with an associated large gauge boson multiplicity.

Method of solution: generation of phase space configurations using an efficient algorithm, coupled to a phenomenological model of the physical processes under study.

Typical running time: 2-3 hours for a $10^{4}$ event simulation (DEC ALPHA 4000), dependent on the process simulated

Unusual features of the program: none. 


\section{Introduction}

It has been known for some time that topologically non-trivial gauge field configurations induce baryon- and lepton-number violating (BLNV) vertices in the electroweak sector of the standard model [1, 2]. More recent investigations of these processes suggest that such processes may be observable at supercollider energies. An extensive review can be found in [3].

However, at present it is not possible to make definite predictions even about the rate of such processes. What is possible is to take the main features of the calculations and use these characteristics to form a phenomenological model of BLNV processes. The aim of such a model is to predict possible experimental avenues that can be explored, with the possibility of improved theoretical calculations in the future allowing the model to be refined. One such model has been presented in [4, 5], and in section 2 we review this model.

In section 3 we describe the implementation of the model as a Monte Carlo simulation, HERBVI. This package simulates hard scattering processes involving BLNV, and has been designed to take into account the very high particle multiplicities associated with BLNV processes. In addition, the formation of the extra baryons or antibaryons from the BLNV process is dealt with.

Finally, in section 4 we make some concluding remarks. In an appendix we describe the use of the HERBVI package by detailing how it interfaces with the general Monte Carlo simulation program HERWIG [6, 7].

\section{Simulating baryon number violation}

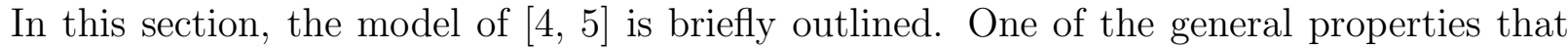
has to be addressed is the parametrically large multiplicity of gauge bosons produced in BLNV interactions [8, 9]. Consequently, we discuss in some detail the method of [10] for the efficient Monte Carlo generation of many-body phase space configurations.

\subsection{General properties}

The general features of instanton-induced BLNV taken into account by HERBVI are:

- Quarks and leptons of all families attached to the BLNV vertex, with total change in baryon and lepton number

$$
\Delta B=\Delta L=-3
$$

- A steeply rising cross section, the rise being cut off at some energy due to unitarity constraints.

- A parametrically large boson multiplicity, $n_{B}$, with a typical value being $n_{B}=30 \sim$ $1 / \alpha_{W}$ 
The BLNV process is assumed to be initiated by a valence quark-quark collision. Thus the reaction is

$$
q+q \rightarrow 7 \bar{q}+3 \bar{l}+n_{B} W(Z)+n_{H} H
$$

where the two incoming quarks and one outgoing antiquark are first-generation, and three outgoing antiquarks of each of the second and third generations are produced. The matrix element is taken to be constant, so that the distributions of produced particles are determined by phase space. Two options for the gauge boson multiplicity $n_{B}$ are provided in the program: either a fixed multiplicity, or a distribution modelled on the leading-order BLNV matrix element. Alternatively, a user-supplied routine can be used. The Higgs boson multiplicity $n_{H}$ is assumed to be tied to that of gauge bosons, as discussed in sect. 3.5 below.

For the energy dependence, a simple step-function of the quark-quark centre-of-mass collision energy $\sqrt{\hat{s}}$ is assumed:

$$
\hat{\sigma}(\sqrt{\hat{s}})=\hat{\sigma}_{0} \theta\left(\sqrt{\hat{s}}-\sqrt{\hat{s}_{0}}\right)
$$

The reasoning behind a model of this form has been discussed in detail in [4]. Again, a user-supplied subroutine may substituted if desired.

The threshold energy parameter $\sqrt{\hat{s}_{0}}$ used in modelling the cross section is expected to be of the order of the characteristic energy of these processes, the sphaleron mass [11], $M_{s p}=\sqrt{6} \pi m_{w} / \alpha_{w} \sim 18 \mathrm{TeV}$. The appropriate value of the cross section parameter $\hat{\sigma}_{0}$ is a matter of great theoretical uncertainty. However, if one wishes to investigate the properties of events rather than their expected number, the magnitude of $\hat{\sigma}_{0}$ is not relevant. For reference, we note that the unitarity bound for S-wave processes is given by

$$
\hat{\sigma}_{U}(\hat{s})=\frac{16 \pi}{\hat{s}}
$$

which is of the order of tens of picobarns at the sphaleron energy $M_{s p}$.

In addition to BLNV events, HERBVI is able to generate the main expected background process, non-perturbative $B$ - and $L$-conserving multi-W production. The reaction is assumed to be

$$
q_{1}+q_{2} \rightarrow q_{3}+q_{4}+n_{B} W(Z)+n_{H} H
$$

This process is also expected to occur at large boson multiplicities, $n_{B} \sim 1 / \alpha_{w}$, with an energy scale $\sim m_{w} / \alpha_{w}$. A detailed discussion, based on the argument that at high energies electroweak theory has a large, approximately constant total inelastic cross section, can be found in [12]. Clearly, these events will be like BLNV ones, but without any fermion number violation. The predominance of boson over fermion production means that the experimental signatures of the two types of events will be similar. It is also expected that the two processes will have similar threshold behaviour and cross sections. In HERBVI they are therefore modelled in the same way, apart from the difference in fermion production.

\subsection{Phase space generation}

The main requirement of a Monte Carlo event generator for simulating BLNV processes is to generate configurations in phase space for high particle multiplicities at high efficiency. 
Therefore, careful consideration of the method used to generate phase space configurations is required.

One approach is to generate $n$-body phase space by factorising the process into $n-1$ two-body decays. This is the method used in, for example, the phase-space generator GENBOD [13]. However, this method results in non-uniform phase space distributions, and in particular becomes very inefficient at high multiplicities. In [14], the efficiency of this method is quoted as being less than $1 \%$ for $n>17$.

The MAMBO algorithm [10] is designed to overcome this problem. The aim is to generate efficiently $N$ momenta $p_{i}^{\mu}$ such that the total momentum

$$
P^{\mu}=\sum_{i=1}^{N} p_{i}^{\mu}
$$

is equal to the total energy and momentum required. In the rest frame of the interaction, this is simply

$$
P^{0}=\sqrt{\hat{s}}, \quad \vec{P}=0 .
$$

In order to develop an efficient algorithm, one may divide the process into two steps. Firstly, $N$ momenta $k_{i}^{\mu}$ are generated, with the only constraint being the mass of each particle, $k_{i}^{2}=m_{i}^{2}$. Obviously, their sum will not satisfy the constraints. The second step is to transform these momenta, using some combination of a Lorentz boost and scaling transformation, so that the resultant momenta $p_{i}^{\mu}$ do satisfy the constraints. Note that we may have different scaling transforms for the space-like and time-like components of the momenta.

The key to the MAMBO algorithm is the scaling transformion used. First, assume that a suitable Lorentz transformation, acting on the $k_{i}^{\mu}$ to produce $q_{i}^{\mu}$, has been performed so that the system is now at rest. Therefore

$$
Q^{\mu}=\sum_{i=1}^{N} q_{i}^{\mu}
$$

such that $\vec{Q}=0$. The scaling transform is now performed by setting

$$
q_{i}^{\mu} \rightarrow x q_{i}^{\mu}, \quad x=\sqrt{\hat{s}} / Q^{0} .
$$

Therefore, the total energy is now $\sqrt{\hat{s}}$ but the particle masses have been rescaled to become $x m_{i}$. The last step is to now find a $\zeta$ such that

$$
\sum_{i=1}^{N}\left(\zeta^{2} \vec{q}_{i}^{2}+m_{i}^{2}\right)^{1 / 2}=\sqrt{\hat{s}}
$$

and then make the replacement

$$
\begin{aligned}
\vec{p}_{i} & =\zeta \vec{q}_{i} \\
p_{i}^{0} & =\sqrt{\zeta^{2} \vec{q}_{i}^{2}+m_{i}^{2}} .
\end{aligned}
$$

The Monte Carlo weight associated with this procedure is

$$
w=\zeta^{3 N-3} \prod_{i=1}^{N}\left(\frac{q_{i}^{0}}{p_{i}^{0}}\right) \frac{\sqrt{\hat{s}}-\sum_{i=1}^{N} x^{2} m_{i}^{2} / q_{i}^{0}}{\sqrt{\hat{s}}-\sum_{i=1}^{N} m_{i}^{2} / p_{i}^{0}} .
$$


It can be shown that $x \leq 1$ implies $w \leq 1$. Therefore, by enforcing the condition $x \leq 1$ and rejecting events with $w$ greater than a uniform random number between 0 and 1 , we can use this algorithm to obtain an unweighted distribution.

The test results described in [10] indicate that, for the typical configurations generated by HERBVI, the efficiency is in the region of $30 \%$ for $n_{B} \sim 30$, falling to $\sim 6 \%$ at the upper end of the range, $n_{B} \sim 120$.

\section{The HERBVI package}

The HERBVI package is designed as an 'add-on' hard process generator for the Monte Carlo event generator HERWIG [6, 7]. The latter program is a general purpose generator for high energy processes involving hadrons. In order to explain how the two packages relate to each other, we now give a quick review of the various stages of event generation in HERWIG.

The first stage is the generation of the hard process under study. This can be either one of the processes already within HERWIG, or alternatively a separate one such as those supplied by HERBVI. The kinematics and parton flavours are generated according to the matrix elements under study, within appropriate phase space limits.

Next, the package simulates the perturbative evolution of the incoming and outgoing partons, by generating parton showers. One of the features of HERWIG is that it correctly treats QCD coherence effects, which lead to an ordering of the emission angles of the partons emitted in the showers. This ordering results in a decrease in the transverse momentum as one moves away from the hard process towards either the initial state beam particles or the final state products. The showering proceeds until either the transverse momentum of emitted partons is below a cut off (final state showers) or when the evolution scale is small enough to be cut off by a scale associated with the structure function (initial state showers).

The end of the showers represents the point where non-perturbative effects lead to hadronisation. HERWIG uses a cluster model [15] to describe this process. Gluons in the parton shower are split non-perturbatively to $q \bar{q}$ pairs, and colour singlet pairs are joined together to form colourless hadronic clusters. These clusters then decay into hadrons, a process simulated using a simple phase space model. The decay of unstable hadrons into stable products is then modelled using particle data tables based on experimental measurements. Heavy quark decays are treated by regarding them as new sources of coherent radiation and repeating the process.

With this sketch of the HERWIG Monte Carlo generator in mind, we now turn to the description of the HERBVI package. Essentially, this models BLNV by generating the appropriate hard process for HERWIG, although the presence of baryon-number violation requires careful treatment when hadronisation is modelled. The package is described by considering various routines within it, in an order corresponding roughly to the order in which they are used by HERWIG. 


\subsection{Initialisation}

HERBVI contains an initialisation procedure designed to make the changing of default values as easy as possible. Without any modification, the package calls the initialisation routines at the start of the generation of the first event.

SUBROUTINE HVHINI - Initialise event generation variables

This routine performs the initialisation of the variables used in event generation. It must be called if certain parameters are changed by the user. The parameters available to the user are, at present, the components of the CKM matrix (stored in the array HVCKM), the cross section parameter HVPLCS, and the limits on the quark-quark subprocess energysquared $\hat{s}$. The lower limit on $\hat{s}$ corresponds to the threshold energy of the model discussed in section 2. The limits for $\hat{s}$ generation are set by the variables SLMIN and SLMAX. These variables are the logarithms of the ratios SLOWER $=\hat{s}_{0} / s$ and SUPPER $=\hat{s}_{\text {max }} / s$, where $s$ is the total centre-of-mass energy squared.

SUBROUTINE HVINIT - Main initialisation routine

This routine is only executed once per program run, and does nothing if called again. All the parameters and flags are set to default values within this routine.

Should the user wish to change any default values at the start of the run, then a call should be made to HVINIT first, and the parameters then changed. A call will then be made automatically to HVHINI at the start of the first event to recalculate derived parameters. If the user wishes to change parameters during a run, another call to HVHINI must be made after the changes.

\subsection{Event generation}

The Monte Carlo program HERWIG uses the HERBVI package for process codes IPROC= 7000 to 7999 . We now examine in detail the various steps the package performs for event generation in this case.

SUBROUTINE HVHBVI - Main interface routine

This routine provides the main event generation interface to HERWIG. Its purpose is to translate HERWIG variables into the appropriate subroutine calls. This is achieved using the HERWIG variables IPROC and GENEV. The first of these determines the type of process to be generated. The values used in HERBVI version 1.0 are listed in Table 1 .

The variable GENEV determines the type of routine to be called. In order to describe its use, we recall first how it functions within the HERWIG package. In HERWIG, a hard process generation routine is called twice. The first call, with GENEV set to .FALSE., requests the generation of an event weight by the hard process routine. The second call,

with GENEV now .TRUE., asks for event generation to be completed and the event record to be written into the standard HEPDATA common block. The HERBVI package is structured in a different manner, with separate routines for weight generation (GENEV=.FALSE.), and 


\begin{tabular}{|c|c|}
\hline \multicolumn{2}{|c|}{ HERBVI process codes } \\
\hline \hline Code & Function \\
\hline $7000-7099$ & BLNV process \\
$7100-7199$ & Multi-W process \\
$7200-7299$ & Random selection \\
\hline
\end{tabular}

Table 1: HERBVI version 1.0 process codes. The random selection is of any code in the range 7000 to 7199 with equal probability.

writing the event data (GENEV=. TRUE.). The event parameters are passed between the two sets of routines using common blocks.

The other function of HVHBVI is a call to HVHINI, in order to initialise variables. This only occurs during the first execution of the routine in each run.

Note that the dummy version of HVHBVI must be deleted from the HERWIG package to allow use of HERBVI.

\subsection{Weight generation}

There are two routines to generate event weights, to cover the BLNV and multi-W cases. We shall discuss the BLNV case first, and then highlight the differences for the $B$ - and $L$-conserving routine.

SUBROUTINE HVHEVT - BLNV weight generation

This routine generates an event weight according to the BLNV model. The first function of the routine is to select the incoming partons, and generate the centre-of-mass energy of the hard process using HVSGEN. Note that the only incoming partons allowed at present are $u$ or $d$ quarks. This restriction is justified since only collisions of protons and/or heavy ions are envisaged, and the high threshold energies of BLNV processes correspond to a high momentum fraction $x$ even at supercollider energies. Consequently valence quark distributions are the only significant source of incoming partons.

The next distribution generated is that of the bosons, accomplished using HVRBOS. Two multiplicity distributions are provided by this routine: a constant or a leading-order type of distribution, selected by means of the logical flag HVCONT (7). These choices correspond to the two cases discussed in [4. Alternatively, it is straightforward for users to provide their own distributions. More details are given in sect. 3.5 below.

The fermions produced in the hard process are now selected according to a simple phase space model. One member of each left-handed doublet has to be chosen. If the masses of the two particles are $m_{1}$ and $m_{2}$, then the probability of choosing particle 1 instead of 2 is

$$
p=\left(1+\frac{m_{2} K_{1}\left(m_{2} \beta\right)}{m_{1} K_{1}\left(m_{1} \beta\right)}\right)^{-1}
$$


for massive particles, where $\beta$ is estimated using

$$
\beta=\frac{3}{2} \frac{n_{B}}{\sqrt{\hat{s}}-n_{B} m_{B}},
$$

and $K_{1}$ is the modified Bessel function of the second kind. For the case $m_{1}=0$, Eq. (13) has to be replaced by

$$
p=\left(1+m_{2} \beta K_{1}\left(m_{2} \beta\right)\right)^{-1} .
$$

A good approximation, used in HERBVI, is to set $p=\frac{1}{2}$ except when selecting from $\bar{t} \bar{b}$ doublets. The error caused by this approximation is negligible, provided $n_{B} m_{B} / \sqrt{\hat{s}}<\frac{1}{2}$.

As pointed out in [1], calculations of fermion number violation by instanton mediated processes deal with gauge-eigenstate down-type quarks, rather than the physical mass eigenstate particles. Therefore, the outgoing quark distribution has to be projected through the CKM matrix. This is achieved by a call to HVHPQM, provided the control flag HVCONT(3) is .TRUE. .

Now that the fermion distribution has been fixed, charge conservation for the whole process is enforced by a second call to HVRBOS, and the structure of the event stored for later use. The $x$ distributions of the two incoming partons can now also be generated, by a call to HVSFUN.

The last step, if requested by changing the value of the flag HVCONT(1) to .FALSE., is to generate the MAMBO weight for the phase space distribution. The anticipated normal use of the package is for an unweighted distribution to be generated during the event data phase of operation, but this can be overridden should the user wish to use a weighted distribution. The main use we foresee for this option is the generation of distributions corresponding to non-trivial matrix elements.

SUBROUTINE HVHMWE - Multi-W weight generation

The only fundamental difference between this routine and HVHEVT is in the nature and number of the outgoing fermions, since $B$ and $L$ are conserved in this case. Again, only $u$ and $d$ quarks are allowed as the incoming partons, $q_{1}$ and $q_{2}$ in Eq. (5), and here the same applies to the outgoing partons, $q_{3}$ and $q_{4}$.

\subsection{Event data}

The event data insertion routines are executed if the event weight has been accepted by HERWIG. As for the weight generation phase, there are separate routines to cover the two types of processes. Again, we describe the BLNV case first and then focus on the differences in the multi-W case.

SUBROUTINE HVHGEN - Generate BLNV event data

This routine writes the event data into the HEPDATA common block, and sets HERWIG variables as appropriate. If the flag HVCONT(1) is .TRUE., its default value, then the MAMBO algorithm is used to generate an unweighted phase space configuration. 
The HERWIG book-keeping is performed by a call to HWEONE, which sets up a $2 \rightarrow 1$ subprocess. The internal HERWIG pointers are then modified to take account of the final state particles. In particular, the colour connection pointers for the fermions are set in such a way that the outgoing light antiquark is connected to the incoming quarks, and the three outgoing quarks of each higher generation are connected to each other, so as to form three distinct colour singlets.

SUBROUTINE HVHMWG - Generate multi-W event data

The main difference between this and HVHGEN is the colour structure of the final state. The colour connections to be made between the partons are now those appropriate to quark-quark elastic scattering. For this purpose, the routine HVETWO is used to set up a $2 \rightarrow 2+n$ process. This is a modification of the HERWIG routine HWETWO, which generates a $2 \rightarrow 2$ process with the correct colour pointers.

\subsection{Generation of distributions}

There are a number of routines within HERBVI to generate the various distributions. They are listed here together for reference. Note that these routines obtain random numbers by calls to random number generation routines within the HERWIG package (named HWR***).

SUBROUTINE HVHPQM - Project quarks through CKM matrix

The elements of the CKM quark mixing matrix are used to project the down-type quarks from their gauge eigenstates onto mass eigenstates. Note that if for any reason the CKM parameters are changed by the user, the initialisation routine HVHINI must be called to reset the parameters used by this routine.

FUNCTION HVRBIN - Binomial distribution

Generation of the binomial distribution

$$
\mathcal{P}(n)=\frac{N !}{n !(N-n) !} p^{n}(1-p)^{N-n} .
$$

The distribution is generated by first generating two uniformly random variables $u$ and $v$, such that $u^{2}<\mathcal{P}(v / u)$, and then setting $n=\operatorname{INT}(v / u)$.

The main use of this routine is in the generation of the $\gamma / Z^{0}$ distribution by splitting the number of neutral bosons $N$ into photons and $Z^{0}$ 's with probabilities $p=\sin ^{2} \theta_{w}$ and $1-p=\cos ^{2} \theta_{w}$ respectively.

SUBROUTINE HVRBOS - Boson number distribution

This routine has two purposes, determined by a flag when it is called. The first is to generate the total number of gauge and Higgs bosons in the final state. The second function is to ensure charge conservation by adjusting the relative numbers of charged bosons. There are three control flags $\operatorname{HVCONT}(7,8,9)$ for this routine.

The flag HVCONT (7) controls the number of gauge bosons, $n_{B}$. If .TRUE., the default, 
then $n_{B}=30$. Otherwise, the number is selected according to a parametrisation of the multiplicity distribution. The default parametrisation is of the form

$$
\mathcal{P}(n, \hat{s})=\exp \left(-\frac{(n+a)^{2}}{b}\right)
$$

where

$$
\begin{aligned}
& a=-21.497 \mathrm{D} 0+0.590 \mathrm{D}-2 \times \frac{\sqrt{\hat{s}}}{\mathrm{GeV}} \\
& b=0.483 \mathrm{D}-1+-0.854 \mathrm{D}-6 \times \frac{\sqrt{\hat{s}}}{\mathrm{GeV}}
\end{aligned}
$$

are parameters found by fitting to the results of phase space calculations using the leading order matrix element for instanton-induced BLNV [16].

The routine can be replaced by a user-supplied version, HURBOS, by setting HVCONT (8) to .FALSE.. Note that both functions of the routine have to be catered for, and that the dummy version of HURBOS must then be deleted from the HERBVI package.

The final flag HVCONT (9) controls the number of Higgs bosons generated. The default is to generate according to a simple model, namely that the probability of generating a Higgs boson is $1 / 16$ of the probability of emitting a gauge boson from the BLNV vertex. This gives a reasonable agreement with the phase space calculations performed in the energy region of interest with $m_{H}=300 \mathrm{GeV}$. Note that the leading-order instanton calculation yields

$$
\begin{aligned}
& \bar{n}_{B} \sim \frac{3}{2} \frac{\pi}{\alpha_{w}}\left(\frac{E}{M_{s p}}\right)^{4 / 3}, \\
& \bar{n}_{H} \sim \frac{3}{32} \frac{\pi}{\alpha_{w}}\left(\frac{E}{M_{s p}}\right)^{2}
\end{aligned}
$$

which, for the region of interest $E \sim M_{s p}$, also predicts the ratio $n_{H} / n_{B} \sim 1 / 16$. The phenomenology of Higgs boson production in BLNV interactions has not yet been considered in detail, essentially because of this expected strong suppression relative to gauge boson production. Setting HVCONT (9)=. FALSE. turns off Higgs boson generation completely.

Once the charge distribution of the particles has been fixed, the numbers of $\gamma$ and $Z^{0}$ bosons are determined using HVRBIN as outlined above.

SUBROUTINE HVSFUN - Parton momentum fraction generation

This routine generates the momentum fractions $x$ of the incoming partons. It requires the centre-of-mass energy of the hard subprocess to be already determined. A weight is generated according to the parton distribution functions of the colliding hadrons.

SUBROUTINE HVSGEN - Hard process energy

Generation of the total centre-of-mass energy $\hat{s}$ for the hard subprocess. The default is to generate $\hat{s}$ according to the distribution $d \hat{s} / \hat{s}$. Setting the flag HVCONT (4) to . FALSE. 
changes this distribution to $d \hat{s} / \hat{s}^{2}$. A user-supplied routine, HUSGEN, can be called instead of HVSGEN if desired by setting HVCONT (6) to .FALSE..

Note that generation of a $d \hat{s} / \hat{s}$ distribution is equivalent to postulating a flat partonlevel cross section. This can be seen by noting that the convolution of the parton level cross section with the proton structure functions,

$$
\sigma(s)=\int_{0}^{1} d x_{1} \int_{0}^{1} \mathrm{~d} x_{2} \int_{0}^{s} \mathrm{~d} \hat{s} f_{1}\left(x_{1}\right) f_{2}\left(x_{2}\right) \delta\left(\hat{s}-x_{1} x_{2} s\right) \hat{\sigma}(\hat{s})
$$

gives

$$
\sigma(s)=\int_{0}^{1} \frac{\mathrm{d} \tau}{\tau} \hat{\sigma}(\tau s) \int_{\tau}^{1} \frac{\mathrm{d} x}{x} x f_{1}(x) \frac{\tau}{x} f_{2}\left(\frac{\tau}{x}\right)
$$

where $\tau=\hat{s} / s$. Specialising to the case of a threshold parton-level cross section

$$
\hat{\sigma}(\hat{s})=\hat{\sigma}_{0} \theta\left(\sqrt{\hat{s}}-\sqrt{\hat{s}_{0}}\right)
$$

we find

$$
\sigma(s)=\hat{\sigma}_{0} \int_{\tau_{0}}^{1} \frac{\mathrm{d} \tau}{\tau} \int_{\tau}^{1} \frac{\mathrm{d} x}{x} x f_{1}(x) \frac{\tau}{x} f_{2}\left(\frac{\tau}{x}\right),
$$

where now $\tau_{0}=\hat{s}_{0} / s$. Therefore, to generate a flat parton-level cross section, we generate $\tau$ according to $d \tau / \tau$ (corresponding to $d \hat{s} / \hat{s}$ ) and $x$ according to $d x / x$, then reweight using the structure functions $z f_{i}(z)$ at $z=x$ and $z=\tau / x$.

Recall that the range of $\hat{s}$ is limited by the parameters SLMIN and SLMAX, which are the logarithms of SLOWER and SUPPER where

$$
\text { SLOWER } \leq \frac{\hat{s}}{s} \leq \text { SUPPER }
$$

The default values correspond to the range

$$
0.24 \leq \frac{\hat{s}}{s} \leq 0.8
$$

which gives $\sqrt{\hat{s}}$ between approximately $50 \%$ and $90 \%$ of the total energy. The upper limit is somewhat arbitrary; the rapid decrease of the structure functions at large $x$ means that the contribution from the upper end of the energy spectrum is negligible. This limit has been introduced to increase the efficiency of the Monte Carlo.

The user can change the variables SLMIN and SLMAX to alter the range of $\hat{s}$ generation at any time, although changing these parameters in the middle of simulation requires a call to HVHINI to enable the derived parameters to be recalculated.

\subsection{Hadron formation}

The colour structure of BLNV processes requires special treatment during the later stages of event processing by HERWIG. As explained above, there are three colour-singlet threequark (antiquark) vertices associated with the process, the first of which comprises the 
two incoming quarks and one outgoing antiquark. The other two correspond to outgoing antiquark triplets.

These vertices do not occur in any baryon-number conserving process, and therefore must be treated by the HERBVI package. However, the set of three partons forming a singlet in the hard subprocess will not necessarily be those forming the 'extra' baryon as a result of BLNV, because the first gluon emitted by any of these partons will carry with it the colour connection to the BLNV vertex. Therefore, the parton cascade within HERWIG must be allowed to occur before the colour connections are made. The point in HERWIG where this occurs is at the start of the clustering phase of the simulation, in the routine HWCFOR.

SUBROUTINE HVCBVI - Find unpaired partons after BLNV

This routine is called at the start of the HERWIG routine HWCFOR, if a BLNV event has been generated. It searches through the event record to locate all partons triplets connected with the BLNV vertices. The end product of the routine is to produce, for each vertex, a quark and associated diquark (or antiquark and antidiquark, as appropriate) that are colour connected.

HERWIG is at present unable to incorporate heavy quarks into diquarks. This means that a vertex such as $\bar{b} \bar{b} \bar{u}$ requires special treatment. The approach used is to form a cluster from two of the BLNV particles, one of which is a heavy antiquark $\bar{Q}$. The cluster is then split, producing an extra $q \bar{q}$ pair. A mesonic colour singlet is formed from $q \bar{Q}$ leaving the light antiquark $\bar{q}$ colour-connected to the BLNV vertex. This approach fails when there is insufficient energy to form the extra $q \bar{q}$ pair. In this case the event is rejected by calling the HERWIG error handler HWWARN with a negative error code, allowing the event to be rejected in a controlled manner. Typically, about $1 \%$ of events have to be rejected in this way.

At the end of this routine, all the BLNV colour connections have been correctly made and the HERWIG package can now complete the process of event generation. Note that, in order to generate BLNV events, the dummy version of HVCBVI must be deleted from HERWIG.

\subsection{Analysis of events}

The HERBVI package contains a routine to aid in the analysis of BLNV events. Whilst this code is not a necessary part of the package, it does aid in the identification of simulated particles direct from the BLNV vertex as opposed to those formed as decay products of other unstable particles.

SUBROUTINE HVANAL - Analyse final state products

The event record is scanned to identify the particles from the BLNV vertex. Particles are flagged as 'primary' from the BLNV vertex if they are either (anti)leptons from the vertex itself, photons from the vertex, or (anti)baryons formed from the colour triplet at the vertex. In addition, some other useful parameters such as the rapidity, pseudo-rapidity 


\begin{tabular}{|l|c|}
\hline \multicolumn{2}{|c|}{ HVADAT contents } \\
\hline \hline Array & Contents \\
\hline IDHNUM & Pointer to entry in HEPEVT \\
PSRFSP & Pseudo-rapidity of particle \\
RAPFSP & Rapidity of particle \\
PTFSP & Transverse momentum of particle \\
IDHFSP & Type of particle (PDG code) \\
ISFSP & TRUE for particle from BLNV vertex \\
\hline
\end{tabular}

Table 2: Contents of the HVADAT common block. Each array listed has one entry for each particle in the final state after the HERWIG simulation phase.

and transverse momentum of each particle are also calculated. The data are stored in the common block COMMON HVADAT.

The contents of HVADAT are complementary to HERWIG output contained in the standard HEPEVT common block, and are listed in Table 2. Note that the file HERBVI10. INC contains the variable declarations for HVADAT. The variable FSPPTR, which is also stored in this common block, contains the total number of final state particles found and processed by HVANAL.

\subsection{Program summary}

For convenience we list here the main routines, control flags and default settings. The routines are listed in Table 3. Other auxiliary routines, not listed here, all have names starting with either HV**** or BVP***. This convention has been followed to avoid clashing with other software. These routines are associated with the internal workings of the HERBVI package and do not concern us here.

The control flags are listed in Table 6 . The default value in all cases, as set by HVINIT, is . TRUE.. The default values of physical parameters are in general taken from the corresponding HERWIG ones. The only exception to this are the limits for the $\hat{s}$ generation, which are given default values by HERBVI as described in section 3.5.

\section{Conclusion}

We have described the various elements of the HERBVI program. This package takes the form of a hard scattering process event generator that interfaces to HERWIG, which is a general-purpose Monte Carlo program for simulating particle interactions involving hadrons.

The phenomenological model which forms the motivation behind the HERBVI package is based upon the features of instanton-induced BLNV calculations, and we have briefly 


\begin{tabular}{|c|c|}
\hline \multicolumn{2}{|c|}{ HERBVI routine names } \\
\hline \hline Routine & Function \\
\hline HVANAL & Initial analysis \\
\hline HVCBVI & Final state clustering \\
HVCBVT & Find hard parton parent \\
\hline HVETWO & $2 \rightarrow 2+n$ hard process \\
\hline HVHBVI & HERWIG interface routine \\
HVHEVT & BLNV weight generation \\
HVHGEN & BLNV event data \\
HVHINI & Initialise derived parameters \\
HVHMWE & Multi-W weight generation \\
HVHMWG & Multi-W event data \\
HVHPQM & CKM matrix projection \\
\hline HVINIT & Main initialisation \\
\hline HVRBIN & Generate binomial distribution \\
HVRBOS & Generate boson distribution \\
\hline HVSFUN & Generate parton $x$ distribution \\
HVSGEN & Generate $\hat{s}$ distribution \\
\hline
\end{tabular}

Table 3: Main HERBVI routines.

\begin{tabular}{|c|c|c|c|}
\hline \multicolumn{3}{|c|}{ HERBVI control flags } \\
\hline \hline Flag & .TRUE. & .FALSE. & Comments \\
\hline HVCONT (1) & Unweighted & User weighted & Phase space generation \\
HVCONT (2) & BLNV & Multi-W & (internal flag) \\
HVCONT (3) & On & Off & CKM matrix projection \\
HVCONT (4) & On & Off & Uniform $\hat{s}$ generation \\
HVCONT (5) & Off & On & Cut-off on $\hat{s}$ generation \\
HVCONT (6) & Off & On & User-supplied $\hat{s}$ generation \\
HVCONT (7) & On & Off & Set $n_{B}=30$ \\
HVCONT (8) & Off & On & User-supplied $n_{B}$ generation \\
HVCONT (9) & On & Off & Allow Higgs production \\
\hline
\end{tabular}

Table 4: Control flags. The default setting for each flag is .TRUE., as set by HVINIT. 
reviewed its features. Of particular importance from an event generation point of view is the parametrically large number of gauge bosons associated with a BLNV event, and we have addressed the issue of efficient phase space generation for such outgoing final states. The simulation of high-multiplicity gauge boson production with conservation of baryon- and lepton- number, which is expected to be the principal background to the BLNV processes considered here, is also implemented.

The violation of baryon number requires particular attention when interfacing to a general simulation package such as HERWIG. Specifically, the formation of the extra (anti)baryons that violate baryon number conservation requires the correct colour connections between partons to be made after the emission of QCD radiation from the partons involved in the hard scattering. The interface between the HERBVI package and HERWIG allows these connections to be made at the appropriate time during event generation.

Finally, we have described the provision that has been made within HERBVI to allow users to provide their own distributions. The distributions contained within the package correspond to the models discussed in [4. The interface to user-supplied distributions has been constructed in a straightforward manner to enable the study of BLNV processes with other parton-level cross sections and boson multiplicity distributions.

\section{Acknowledgement}

We are most grateful to Andreas Ringwald for many helpful discussions and suggestions during the development of the program.

\section{Appendix A: Installing HERBVI}

The HERBVI package is designed to be used with the HERWIG Monte Carlo simulation package. Versions 5.7 and later of HERWIG contain hooks that allow the two packages to link together in a relatively simple manner. There are two steps required to link the packages.

- The dummy subroutines HVCBVI and HVHBVI must be deleted from the HERWIG package. Failure to do this will cause the program to terminate with an error message when the first attempt to generate a BLNV event is made.

- The parameters NMXHEP, NMXPAR, MODMAX and NMXJET must be increased from their default values. The default values are set in the file HERWIGxy. INC, where $\mathrm{x} . \mathrm{y}$ is the program version number, and the (recommended) new values are shown in Table 5 . If this change is not made then HERWIG will run out of internal working space with unpredictable results.

The first change can be made by deleting the dummy routines from the HERWIG source code. The second change must be made by editing the parameter settings in the 


\begin{tabular}{|c|r|r|}
\hline \multicolumn{3}{|c|}{ Parameter settings for HERBVI } \\
\hline \hline Parameter & Default & HERBVI \\
\hline NMXHEP & 2000 & 15000 \\
NMXPAR & 500 & 2000 \\
MODMAX & 5 & 200 \\
NMXJET & 200 & 500 \\
\hline
\end{tabular}

Table 5: Recommended HERWIG parameter settings for use with the HERBVI package.

HERWIGxy. INC file. The changes in these parameters are required to give the HERWIG package enough working space to process the high-multiplicity BLNV events. Note that the formatting of printed output from HERWIG is automatically changed for NMXHEP values greater than 9999, in order to ensure that the 80-column output is readable.

It should be stressed that, after the above changes have been made, the HERWIG package must be recompiled to reflect the change in size of the common blocks.

Finally, we remind the reader that to use HERBVI with user-defined distributions (HVCONT (6) and/or HVCONT (8)=. FALSE.), the appropriate subroutines HUSGEN and/or HURBOS must be supplied by the user and the corresponding dummy subroutines deleted from the HERBVI package.

\section{Appendix B: Test run output}

The output of the HERBVI package, after processing by the HERWIG program, is contained as a list of final state hadrons and leptons within the standard HEPEVT common block. As there are typically $\sim 10^{3-4}$ particles produced per HERBVI event, is is not practical to show one of these events here. Instead, we show the output of the test program supplied with the HERBVI package. This program generates 1000 events, with a total collider energy of $40 \mathrm{TeV}$, using the IPROC=7200 option.

The implementation of random number generators is, generally speaking, not machineindependent due to numerical precision effects. Therefore, one should expect to get similar, but not necessarily identical, results when comparing the output of HERBVI runs from different computer architectures.

$\begin{array}{lr}\text { OUTPUT ON ELEMENTARY PROCESS } \\ \text { NUMBER OF EVENTS }= & 992 \\ \text { NUMBER OF WEIGHTS }= & 52930 \\ \text { MEAN VALUE OF WGT }= & 1.6247 \mathrm{E}-04 \\ \text { RMS SPREAD IN WGT }= & 6.0487 \mathrm{E}-04 \\ \text { ACTUAL MAX WEIGHT }= & 7.7377 \mathrm{E}-03 \\ \text { ASSUMED MAX WEIGHT }= & 8.2604 \mathrm{E}-03\end{array}$


PROCESS CODE IPROC $=\quad 7200$

CROSS SECTION $(\mathrm{PB})=0.1625$

ERROR IN C-S $(P B)=2.6291 \mathrm{E}-03$

EFFICIENCY PERCENT $=1.967$

HERBVI parameters

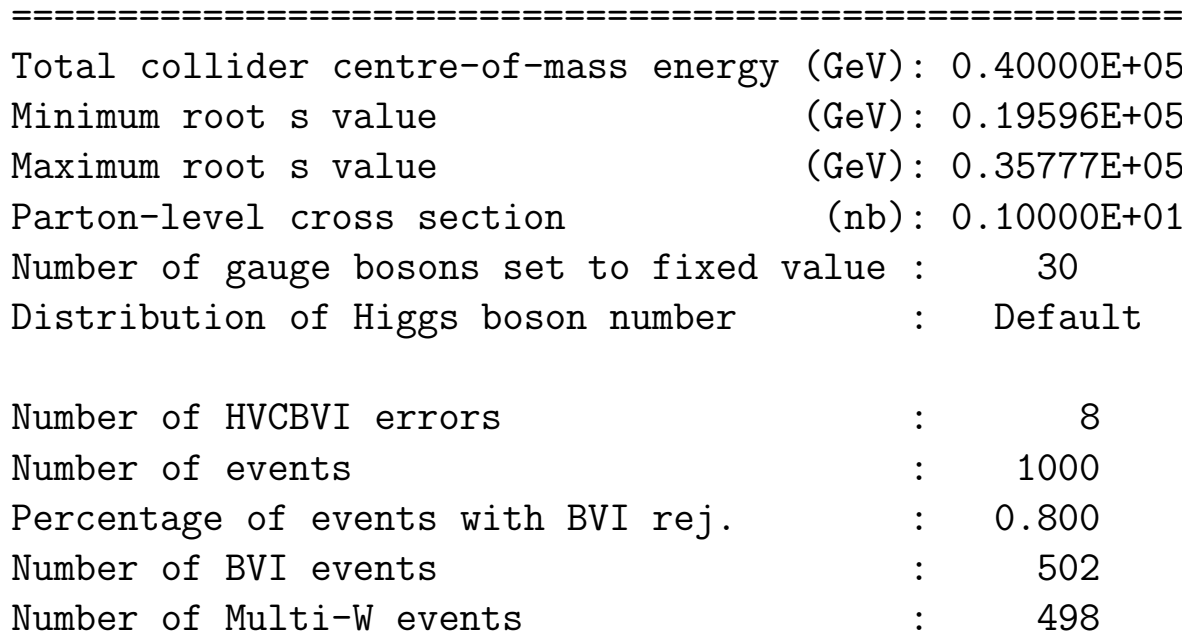

\section{References}

[1] G. 't Hooft, Phys. Rev. D14 (1976) 3432.

[2] G. 't Hooft, Phys. Rev. Lett. 37 (1976) 8.

[3] M. Mattis, Phys. Rev. 214 (1992) 159.

[4] M.J. Gibbs, A. Ringwald, B.R. Webber and J.T. Zadrozny, CERN preprint CERNTH-7090/93, Cavendish preprint Cavendish-HEP-93/6, to appear in Zeit. Phys. C.

[5] M.J. Gibbs, Cavendish preprint Cavendish-HEP-94/5, to appear in Phys. Lett. B.

[6] G. Marchesini and B.R. Webber, Nucl. Phys. B310 (1988) 461.

[7] G. Marchesini, B.R. Webber, G. Abbiendi, I.G. Knowles, M.H. Seymour and L. Stanco, Comp. Phys. Comm. 67 (1992) 465.

[8] A. Ringwald, Nucl. Phys. B330 (1990) 1.

[9] O. Espinoza, Nucl. Phys. B343 (1990) 310.

[10] R. Kleiss and W.J. Stirling, Nucl. Phys. B385 (1992) 413.

[11] F. Klinkhamer and N. Manton, Phys. Rev. D30 (1984) 2212.

[12] A. Ringwald, F. Schrempp and C. Wetterich, Nucl. Phys. B365 (1991) 3. 
[13] F. James, CERN Program Library; CERN 68-15 (1968).

[14] W.J. Stirling, R. Kleiss and S.D. Ellis, Comp. Phys. Comm. 40 (1986) 359.

[15] B.R. Webber, Nucl. Phys. B238 (1984) 492.

[16] G. Farrar and R. Meng, Phys. Rev. Lett. 65 (1990) 3377. 